\title{
Microwave-Assisted Linear Approach Toward Highly Substituted Benzo[d]oxazol-5-yl-1H-benzo[d]imidazole on Ionic Liquid Support
}

\author{
Kaushik Chanda, Barnali Maiti, Chih-Chung Tseng, and Chung-Ming Sun* \\ Laboratory of Combinatorial Drug Design, Department of Applied Chemistry, National Chiao-Tung University, Hsinchu 300-10, \\ Taiwan
}

\section{Supporting Information}

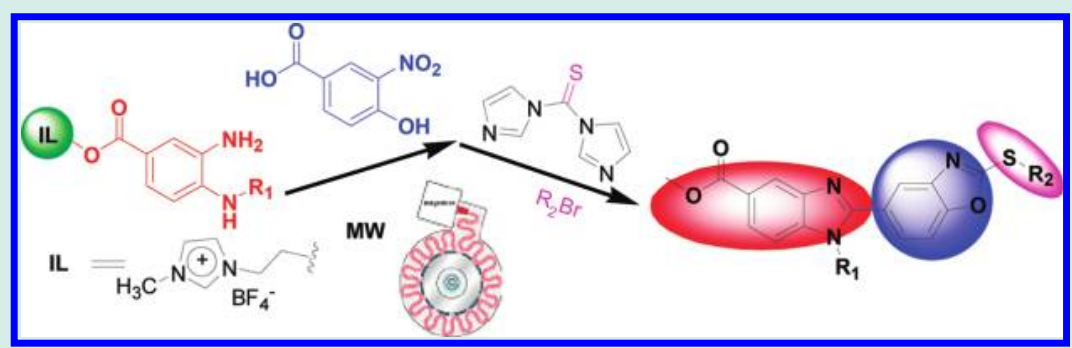

ABSTRACT: A novel and efficient diversity-oriented synthetic approach was employed to access the benzo $[d]$ oxazol-5-yl-1Hbenzo $[d]$ imidazole on ionic liquid support, which helps to absorb microwave irradiation. In this paper, we successfully coupled 4hydroxy-3-nitrobenzoic acid onto ionic liquid-immobilized o-phenylenediamine, which subsequently underwent an acid mediated, ring closure reaction leading to benzimidazole derivatives. After hydrogenation of the nitro group to an amine, the resulting ionic liquid conjugate was reacted with 1,1-thiocarbonyldiimidazols to yield an ionic liquid tagged-benzoxazol. Final skeletal diversity of the present scaffold was further achieved by S-alkylation with alkyl and aryl bromides. The benzo[d]oxazol-5yl-1H-benzo $[d]$ imidazole was finally cleaved smoothly from the ionic liquid support with sodium methoxide in methanol under microwave irradiation. This methodology has provided access to a small, diverse library by straightforward and simple operations and could be applied readily in various drug discovery programs.

KEYWORDS: microwave-assisted, benzo[d]oxazol-5-yl-1H-benzo[d]imidazole, ionic liquid-immobilized

\section{INTRODUCTION}

With the introduction of chemical genetics, the study of important biological targets has basically relied on the extensive use of small molecules. ${ }^{1}$ The design and proficient assembly of molecularly diverse heterocyclic libraries are key for medicinal chemists. The small molecule focused libraries have been used to investigate particular targets that could perturb the specific function of the targets in a systematic manner with the generation of information for further biomedical research., Because of the increasing demand of novel small molecules for high-throughput screening, numerous synthetic methodologies in combinatorial chemistry including solid support and solution phase strategies have been developed through multidisciplinary approaches. ${ }^{4}$ Drawbacks in solid-phase organic synthesis (SPOS) has encouraged research in ionic liquid phase organic synthesis. ${ }^{5}$ It has been increasingly feasible to construct the complex molecular architectures through multistep synthetic sequence on the basis of ever expanding range of ionic liquids. Recently, ionic liquids (ILs) have received considerable attention as environmentally benign reaction medium for chemical reactions, including some enzymatic reactions in organic chemistry. ${ }^{6}$ The most prominent feature of ionic liquids is their tunable solubility and phase separation from organic to aqueous phase depending on the choice of cations and anions. Because of this property, ionic liquids have emerged as a soluble support for small molecule organic synthesis by retaining the advantages of product isolation and purification of solid-phase synthesis along with benefits of traditional solution phase chemistry. ${ }^{7}$ The organic substrate can be anchored on the ionic liquid support initially while carrying out the planned multistep synthesis without detaching the ionic liquid for monitoring reaction progress at every stage. Since the desired synthetic sequence can be carried out in a homogeneous phase, the excess amounts of reagents and side products are removed simply by washing with less polar organic solvents. Once the desired transformations have been accomplished, the ionic liquid support can be removed to release the target libraries. Finally, the liberated ionic liquid can be recycled and resubjected to other ionic liquid organic synthetic. The importance of the ionic liquid phase organic synthesis has been effectively utilized for the diversity-oriented synthesis to synthesize small molecules and peptides. ${ }^{8}$ To facilitate the design of novel bioactive compounds, the combination of different pharmacophores into one framework can lead to new hybrid molecules with better binding opportunities inside target active sites. By simply changing

Received: November 9, 2011

Revised: January 11, 2012

Published: January 20, 2012 
the substitution pattern, new scaffolds obtained from the combinatorial techniques have been able to address the different targets. In view of our continuous interests on benzimidazole and benzoxazols moieties, the combination of two pharmacophore, benzoxazol and benzimidazole moieties into one molecule may offer interesting biological profiles. ${ }^{9}$

To demonstrate the diversity-oriented synthesis on an ionicliquid support, benzo $[d]$ oxazol-5-yl-1H-benzo $[d]$ imidazoles were chosen as the target compounds that have very distinct biological activity as therapeutic agents. Benzimidazoles are an important class of heterocyclic compound in diverse therapeutic areas including antiulcer, antihypertensive, antiviral, anticancer, antifungal, antitubercular, antiallergic, antihistaminic, antioxidant, and antimicrobial activities. ${ }^{10}$ Prominently, the benzoxazol and its thio-analogues are potentilal KDR inhibitors 1, cyclooxygenase inhibitors 2, selective $5-\mathrm{HT}_{1 \mathrm{~A}}$ serotonin receptor ligands 3 (Figure 1). ${ }^{11}$

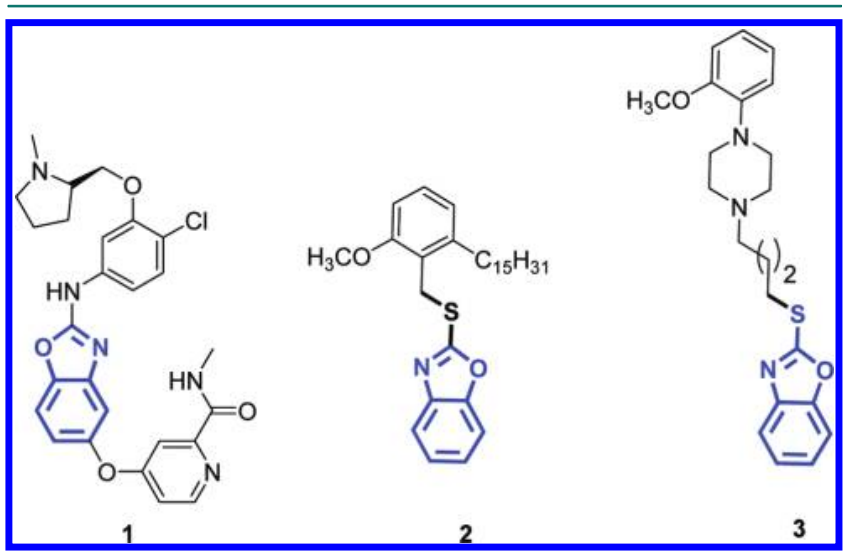

Figure 1. Biologically active benzoxazols derivatives.

The existing synthetic strategies to benzoxazols mainly rely on solid-phase synthetic approaches. ${ }^{12}$ In view of the potential bioactivity of benzoxazol cores and their limited synthetic scope, we report an application of microwave irradiation for parallel synthesis of benzo[d]oxazol-5-yl-1H-benzo[d]imidazoles using ionic liquid as a phase tag, which is envisaged to provide a homogeneous media for better reaction kinetics and enable direct monitoring of reactions not achieved by solidphase approaches.

\section{RESULTS AND DISCUSSIONS}

Our synthetic strategy to develop the intended molecular framework of the benzo[ $d]$ oxazol-5-yl- $1 H$-benzo[ $d]$ imidazole is depicted in Scheme 1. The planned strategy commenced with the application of the ionic liquid 1-(2-hydroxyethyl)-3methylimidazolium tetrafluoroborate ([hydemim $]\left[\mathrm{BF}_{4}\right]$ ) equipped with a hydroxyl group linker as a soluble support. Accordingly, the ionic liquid-bounded ortho-phenylenediamine 4 was synthesized from 4-fluoro-3-nitrobenzoic acid with builtin structural diversity $\left(\mathrm{R}_{1}\right)$ through a three-step protocol. ${ }^{13} \mathrm{We}$ envisioned to apply amide coupling and subsequent cyclization at the primary amine functionality on ionic liquid conjugate 4 leading to an aryl imidazole skeleton, which can be further elaborated on benzoxazol 9 and S-alkylated benzoxazol 10 as downstream products.

The ionic liquid bound compound 4 was then $\mathrm{N}$-acylated at the primary amine with 4-hydroxy-3-nitrobenzoic acid 5 as shown in Scheme 2. Accordingly, the anilide conjugates 6 were obtained by the condensation of 4-hydroxy-3-nitrobenzoic acid 5 with ionic liquid conjugates 4 via $\mathrm{DCC} / \mathrm{HOBt}$ activation for $12 \mathrm{~h}$ under refluxing $N, N^{\prime}$-dimethylformamide (DMF). However, the use of microwave irradiation at $160{ }^{\circ} \mathrm{C}$ dramatically reduced the reaction time to $10 \mathrm{~min}$.

By taking the advantage of the solubility profile of ionic liquids, we further purified the reaction mixture by precipitation with ether. For the construction of benzimidazole ring, anilide conjugates 6 were subjected to acid-catalyzed cyclization in presence of $10 \%$ trifluoroacetic acid under refluxing $\mathrm{CH}_{3} \mathrm{CN}$ for $5 \mathrm{~h}$. To facilitate the synthesis of the target compounds, we applied the microwave irradiation $\left(140{ }^{\circ} \mathrm{C}\right)$ for the same reaction to complete in $5 \mathrm{~min}$ to yield 2 -arylbenzimidazole conjugates 7 . Reduction of the nitro group on conjugate 7 was achieved by palladium on activated charcoal (10\%) with ammonium formate in methanol under microwave heating at $90{ }^{\circ} \mathrm{C}$ for $5 \mathrm{~min}$. The formation of the amine conjugates 8 could also be confirmed by color change of the reaction mixture from yellow to blue along with the change in the chemical shifts of aromatic regions in ${ }^{1} \mathrm{H}$ NMR spectra of the crude products. The reaction mixture was precipitated with ether to obtain the conjugates 8. Our main goal is to construct benzoxazole ring in conjunction with additional diversity through simple operations. The elaboration of ionic liquid bound 3-amino-4hydroxy benzimidazolyl derivatives $\mathbf{8}$ to the desired core structure requires a one carbon synthon. We envisioned thiocarbonyl diimidazole (TCDI) to be suitable as a one-

Scheme 1. Synthetic Strategy for the Benzo $[d]$ oxazol-5-yl-1H-benzo $[d]$ imidazole 10

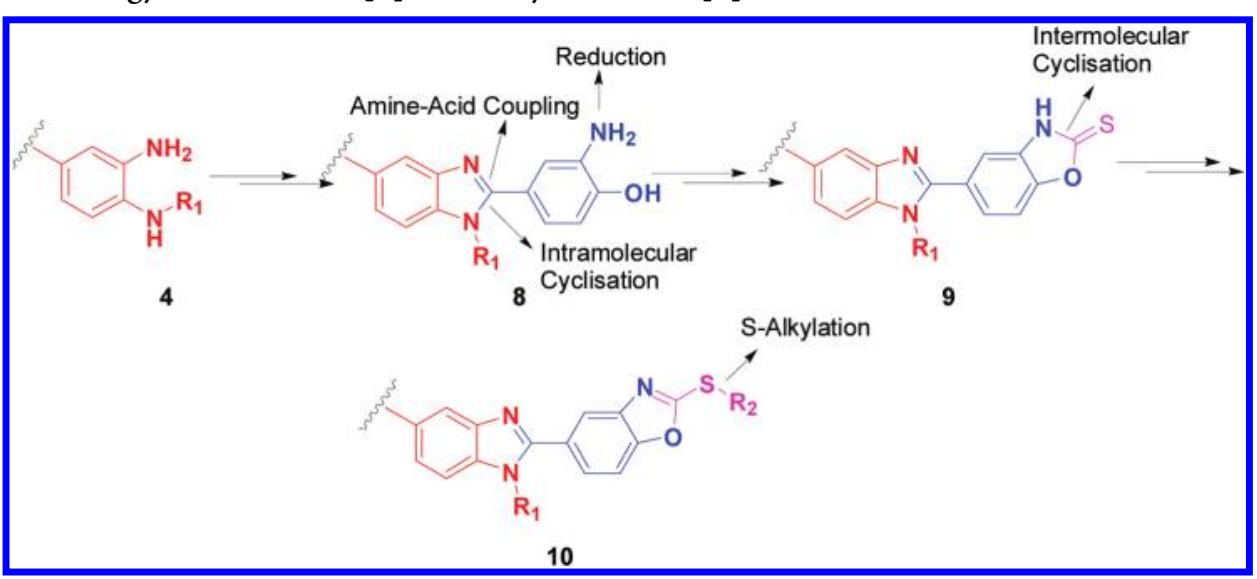


Scheme 2. General Strategy of Microwave-Assisted Synthesis of Benzimidazolyl Benzoxazols on Ionic Liquid Support

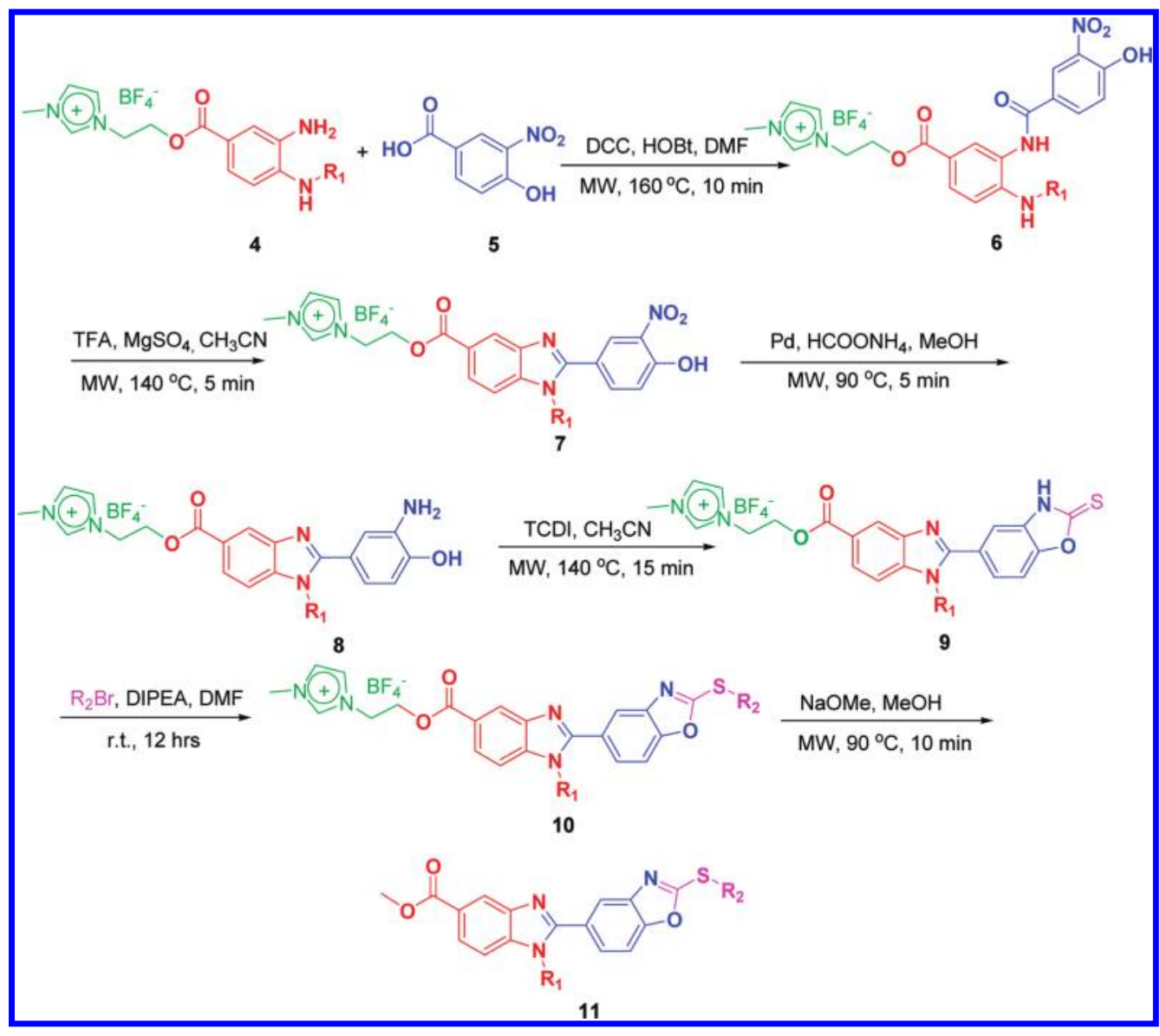

carbon electrophile to fulfill our desire for the construction of benzoxazole ring at this penultimate step. The amine conjugates 8 were condensed with thiocarbonyl diimidazole in anhydrous $\mathrm{CH}_{3} \mathrm{CN}$ under microwave irradiation at $140{ }^{\circ} \mathrm{C}$ to furnish the ionic liquid bound benzoxazole conjugates 10 in 15 min. In comparison, the same transformation required $6 \mathrm{~h}$ under conventional refluxing conditions to reach full conversion. The S-alkylation of ionic liquid conjugates benzoxazolin-2-thione 9 is the second access point to extend the chemical diversity. Literature survey revealed that $S$ alkylation of such heterocyclic system can occur under mild alkaline conditions, which were employed to synthesize a number of biologically active sulfides. ${ }^{14}$ In principle, this alkylation could take place at $\mathrm{N}$ - or S-terminus to afford a mixture of products. To our delight, subjection of benzoxazolin2-thione 9 with various alkyl bromides in presence of diisopropylethylamine at room temperature led to S-alkylated conjugates 10 in good yields. The bis-heterocyclic molecules consisting of benzimidazolyl benzoxazole were cleaved off from ionic liquid support using 0.1 M solution of $\mathrm{NaOMe}$ in $\mathrm{MeOH}$ at room temperature for $12 \mathrm{~h}$. The same cleavage exercise was complete in 10-15 min under microwave conditions. Ionic liquid support was then removed by filtration after precipitation with ether and the final compounds were quantified by HPLC analysis indicating 76-99\% crude purity of title compounds. Purification the resulting crude products by column chromatography afforded the thio analogs of bis-benzimidazolyl benzoxazole derivatives $\mathbf{1 1}$ in good overall yields. By manipulation of reaction sequence, a variety of 2-sulphanylated benzo[d] oxazol-5-yl-1H-benzo[d]imidazole 11 were rapidly obtained (Table 1).

However, to confirm the formation of $\mathrm{N}$-or S-alkylated product, we cleaved the ionic liquid bound benzimidazole linked benzoxazolin-2-thione $9\{2\}$ using $0.1 \mathrm{M}$ solution of $\mathrm{NaOMe}$ in $\mathrm{MeOH}$ under the microwave condition $\left(90{ }^{\circ} \mathrm{C}\right)$ for 10 min to obtain the intermediate $\mathbf{A}$ and characterized by the study of ${ }^{1} \mathrm{H}$ and ${ }^{13} \mathrm{C}$ NMR spectroscopy and LR, HR spectra (see Supporting Information). Exclusive formation of $S$ alkylated product has been concluded by examination of its ${ }^{13} \mathrm{C}$ NMR spectrum revealing the resonance signal at 181.9 ppm representing the $\mathrm{C}=\mathrm{S}$ fragment in benzoxazolin-2-thione (A) upfield shifted to $168.0 \mathrm{ppm}$ after been alkylated with cinnamyl bromide (Figure 2).

Unlike solid-support approaches, the main advantage of using an ionic liquid as support was its direct reaction monitoring facility by standard analytical techniques, such as ${ }^{1} \mathrm{H},{ }^{13} \mathrm{C} \mathrm{NMR}$, and mass spectroscopy without cleaving the ionic liquid tag. Key ionic liquid-tagged intermediates en route toward the final target molecule were examined by ${ }^{1} \mathrm{H}$ NMR spectroscopy to demonstrate this advantageous in reaction monitoring and structural characterization of products (see Supporting Information).

Unambiguous structural assignment of nonplanar thio analogue of benzimidazolyl benzoxazol skeleton 11 was confirmed by signal crystal X-ray diffraction in the case of compound $11\{2,1\}$ (Figure 3). ${ }^{15}$

The crystal structure reveals the biheterocyclic configured with nonplanarity in the core skeleton. Moreover, it has been found that the benzoxazole moiety is located perpendicular to 
Table 1. Synthesis of Thio Analogues of Benzimidazolyl Benzoxazol Derivatives 11 using Microwave Irradiation on Ionic Liquid Support

\begin{tabular}{|c|c|c|c|c|c|c|}
\hline Entry & $\mathrm{R}_{1} \mathrm{NH}_{2}\{1-6\}$ & $\mathrm{R}_{2} \mathrm{Br}\{1-5\}$ & Products & Yield $^{2}$ & Purity ${ }^{b}$ & LRMS $^{C}$ \\
\hline 1 & & & $11\{1,1\}$ & 79 & 77 & 550 \\
\hline 2 & & & $11\{2,1\}$ & 81 & 95 & 498 \\
\hline 3 & & & $11\{3,2\}$ & 90 & 98 & 518 \\
\hline 4 & & & $11\{3,3\}$ & 92 & 91 & 450 \\
\hline 5 & & & $11\{2,2\}$ & 89 & 99 & 518 \\
\hline 6 & & & $11\{1,2\}$ & 91 & 95 & 570 \\
\hline 7 & & & $11\{1,3\}$ & 95 & 97 & 502 \\
\hline 8 & & & $11\{4,4\}$ & 88 & 95 & 434 \\
\hline 9 & & & $11\{4,2\}$ & 84 & 97 & 530 \\
\hline 10 & & & $11\{5,5\}$ & 89 & 87 & 511 \\
\hline 11 & & & $11\{5,4\}$ & 88 & 86 & 461 \\
\hline 12 & & & $11\{5,2\}$ & 90 & 93 & 557 \\
\hline 13 & & & $11\{6,4\}$ & 89 & 92 & 446 \\
\hline 14 & & & $11\{6,2\}$ & 80 & 76 & 542 \\
\hline 15 & $\mathrm{H}_{2}$ & & $11\{2,4\}$ & 94 & 97 & 422 \\
\hline 16 & & & $11\{2,5\}$ & 90 & 92 & 472 \\
\hline
\end{tabular}

${ }^{a}$ Isolated yields determined on weight of purified samples (\%). ${ }^{b}$ Determined by HPLC analysis (UV detection at $254 \mathrm{~nm}$ ) of the crude product (\%). ${ }^{c}$ LRMS were detected with ESI ionization source.

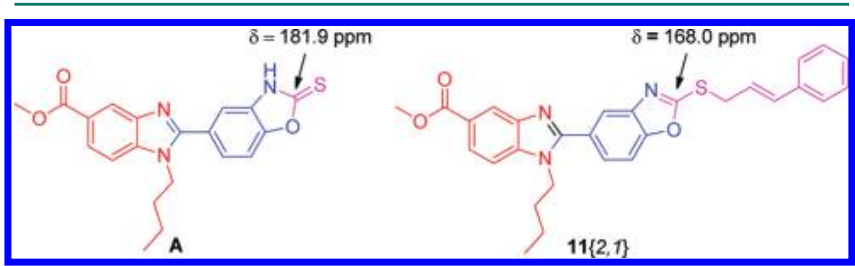

Figure 2. Comparison of ${ }^{13} \mathrm{C}$ NMR chemical shifts between compounds $\mathbf{A}$ and $\mathbf{1 1}\{2,1\}$ at the indicated carbon.

the plane occupied by the benzoimidazole moiety, whereas cinnamyl ring remains perpendicular to the plane which was confirmed from its 3D model.

\section{CONCLUSIONS}

In summary, we have developed an efficient microwave-assisted synthesis toward a bis-heterocycle library of benzimidazolyl benzoxazols on ionic liquid support. Using this strategy, we have synthesized a wide variety of functionalized derivatives exploiting the readily available primary amine and substituted bromide. The construction of benzoxazols over benzimidazole involves the sequential amine-acid coupling, cyclization, reduction and final cyclization reactions with 1,1-thiocarbonyldiimidazole. Final diversification was achieved via Salkylation with substituted bromides without cleavage of the ester bond at the ionic liquid attached site under microwave harsh conditions. This multistep synthetic strategy allowed us to obtain IL-attached intermediates with low levels of impurities by simple precipitation. Furthermore, it should be noted that the thio analogs of benzo $[d]$ oxazol-5-yl- $1 \mathrm{H}$ benzo $[d]$ imidazole are virtually unprecedented in literature. Coupled with the use of inexpensive starting materials, operational simplicity, and short reaction time under microwave irradiation on ionic liquid support makes the method potentially scaleable and should be applied to prepare a wide range of analogues.

\section{EXPERIMENTAL SECTION}

General Procedure for the Preparation of Ionic Liquid Bound 3-(4-Hydroxy-3-nitrobenzamido)-4-(substituted amino) Carboxylates 6 . To a solution of $N, N^{\prime}$-dicyclohexylcarbodiimide (DCC) $(710 \mathrm{mg}, 3.45 \mathrm{mmol}, 1.4$ equiv) in $N, N^{\prime}$-dimethylformamide (DMF) was added 4-hydroxy-3nitrobenzoic acid 5 (585 mg, $3.20 \mathrm{mmol}, 1.3$ equiv) and 1hydroxybenzotriazole (HOBt) $(432 \mathrm{mg}, 3.20 \mathrm{mmol}, 3.20$ equiv) in a sequential order. The resulting slurry was stirred for $5 \mathrm{~min}$ at room temperature, and then ionic liquid (IL) anchored o-phenylene diammine $4(1.0 \mathrm{~g}, 2.46 \mathrm{mmol}, 1.0$ equiv) in DMF $(5 \mathrm{~mL})$ was added. The reaction mixtures were subsequently heated with stirring in a $10 \mathrm{~mL}$ microwave process vial at $160{ }^{\circ} \mathrm{C}$ for $10 \mathrm{~min}$ to obtain the ionic liquid conjugate 6. After completion of the reaction, the suspension byproducts were filtered through filter paper. The reaction mixture was precipitated by slow addition of cold ether and precipitated amide conjugates 6 were filtered through fritted funnel. The crude product was washed in succession with ether $(100 \mathrm{~mL} \times 3)$ to remove the undesired impurity and dried for further steps.

General Procedure for the Preparation of Ionic Liquid Bound 2-(4-Hydroxy-3-nitrophenyl)-1-alkyl-1H-benzo[d] imidazole Carboxylates 7. To a solution of ionic liquidbound 3-(4-hydroxy-3-nitrobenzamido)-4-(substituted amino) carboxylates 6 in acetonitrile, trifluoroacetic acid $(0.5 \mathrm{~mL})$ and $\mathrm{MgSO}_{4}(500 \mathrm{mg}$ ) were added, and the mixture was subsequently heated with stirring in a $10 \mathrm{~mL}$ microwave process vial at $140{ }^{\circ} \mathrm{C}$ for $5 \mathrm{~min}$. After completion of the reaction, $\mathrm{MgSO}_{4}$ was removed through Celite. The reaction mixtures were precipitated by slow addition of excess of cold ether $(100 \mathrm{~mL})$, and filtered through a fritted funnel to obtain the ionic liquid 2-(4-hydroxy-3-nitrophenyl)-1-alkyl-1H-benzo[d]imidazole carboxylates 7 in high purity.

General Procedure for the Preparation of Ionic liquid Polymer Bound 2-(3-Amino-4-hydroxyphenyl)-1-alkyl$1 \mathrm{H}$-benzo[d]imidazole Carboxylates 8. To a solution of 7 in methanol, $\mathrm{Pd}$ on charcoal $(1.30 \mathrm{~g}, 12.65 \mathrm{mmol}, 5.0$ equiv.) and ammonium formate $(1.12 \mathrm{~g}, 17.72 \mathrm{mmol}$, and 7.0 equiv) were added. The reaction mixture was subsequently heated with stirring in a $10 \mathrm{~mL}$ microwave process vial $\left(90^{\circ} \mathrm{C}\right)$ for 5 $\mathrm{min}$ in the appropriate mode of pressure and temperature to complete reduction of nitro group which was evident from color change (yellow to greenish blue). After completion, the reaction mixtures were then subjected to centrifugation for removal of Pd on charcoal, and the supernatant liquid was concentrated by rotary evaporation to remove methanol. Acetonitrile $(10 \mathrm{~mL})$ was then added to salt out ammonium 


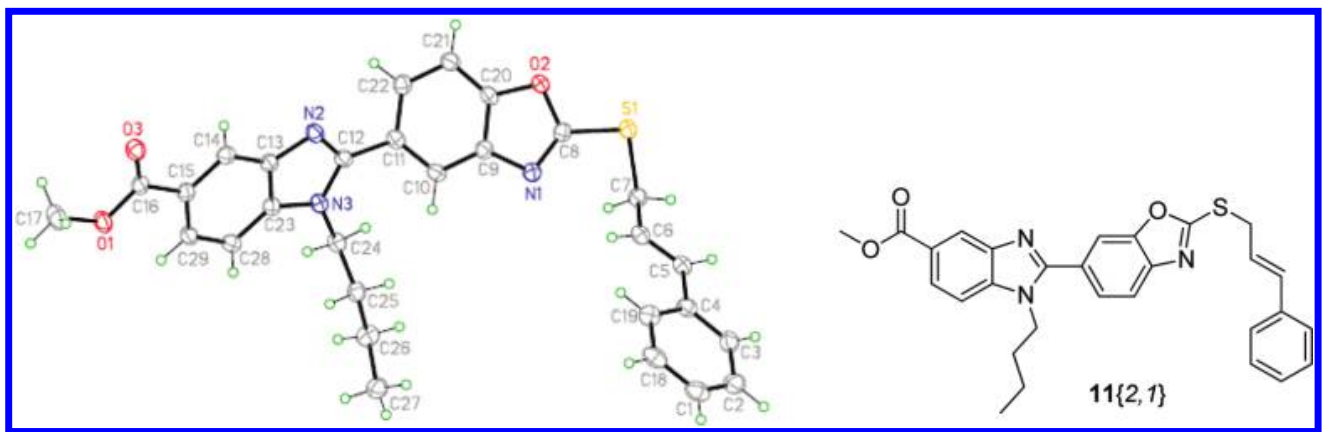

Figure 3. ORTEP diagram of 1-Butyl-2-(2-(cinnamylthio)benzo[d]oxazol-5-yl)-1-1H-benzo[d]imidazole-5-carboxylic acid methyl ester 11 $\{2,1\}$ (Table 1, entry 2 ).

formate. The reaction mixtures were filtered through fritted funnel to remove ammonium formate to obtain the ionic liquid bound 2-(3-Amino-4-hydroxyphenyl)-1-alkyl-1H-benzo[d]imidazole carboxylates 8 .

General Procedure for the Preparation of Ionic liquid Bound 2-(2-Mercaptobenzo[d]oxazol-5-yl)-1-alkyl-1Hbenzo[d]imidazole Carboxylates 9. To a stirred solution of ionic liquid bound 2-(3-amino-4-hydroxyphenyl)-1-alkyl-1 $\mathrm{H}$ benzo[d]imidazole carboxylates conjugates 8 in $\mathrm{CH}_{3} \mathrm{CN}$ (5 $\mathrm{mL}$ ), 1,1-thiocarbonyldiimidazole $(663 \mathrm{mg}, 3.73 \mathrm{mmol}, 1.5$ equiv) was added. The reaction mixtures were subsequently heated with stirring in a $10 \mathrm{~mL}$ microwave process vial (140 ${ }^{\circ} \mathrm{C}$ ) for $5 \mathrm{~min}$ in the appropriate mode of pressure and temperature for complete cyclization. After completion, the reaction mixture was precipitated by slow addition of excess of cold ether $(100 \mathrm{~mL})$ and filtered through a fritted funnel to obtain the ionic liquid 2-(2-mercaptobenzo[d] oxazol-5-yl)-1alkyl-1H-benzo $[d]$ imidazole carboxylates 9 in high purity.

General Procedure for the Preparation of lonic liquid Bound 2-(2-(alkylthio)benzo[d]oxazol-5-yl)-1-alkyl-1 Hbenzo[d]imidazole carboxylates 10 . To a stirred solution of ionic liquid bound 2-(2-(alkylthio)benzo[d]oxazol-5-yl)-1alkyl-1H-benzo[d]imidazole carboxylates conjugates 9 in $N, N^{\prime}$ dimethylformamide (5 mL) (DMF), N,N-diisopropylethyl amine (480 mg, $3.71 \mathrm{mmol}, 1.5$ equiv) and various alkyl bromides ( $3.71 \mathrm{mmol}, 1.5$ equiv) were added. The reaction mixtures were subsequently stirred for $12 \mathrm{~h}$ under room temperature for complete alkylation. After completion, the reaction mixture were precipitated by slow addition of excess of cold ether $(100 \mathrm{~mL})$ and filtered through a fritted funnel to obtain the ionic liquid 2-(2-(alkylthio)benzo[d] oxazol-5-yl)-1alkyl-1H-benzo $[d]$ imidazole carboxylates $\mathbf{1 0}$ in high purity.

General Procedure for the Cleavage of lonic liquid Bound 2-(2-(Alkylthio)benzo[d]oxazol-5-yl)-1-alkyl-1 Hbenzo[d]imidazole Carboxylate 11 . To a solution of conjugates 10 in methanol $(20 \mathrm{~mL}), \mathrm{NaOMe}(100 \mathrm{mg})$ was added and irradiated under pressured microwave irradiation at $\left(90^{\circ} \mathrm{C}\right.$ ) for $12 \mathrm{~min}$. After completion of the reaction, the crude product was precipitated with excess of cold ether $(100 \mathrm{~mL})$, the ionic liquid was filtered off and subjected to evaporation. The residue was dried under vacuum, and subjected to crude HPLC analysis with UV detection at $\lambda=254 \mathrm{~nm}$ (column, sphereclone $5 \mu \mathrm{Si}(250 \times 4.6 \mathrm{~mm})$; gradient, $35 \%$ ethyl acetate in hexane; flow rate, $1 \mathrm{~mL} / \mathrm{min}$.). The purity of crude products was as indicated in Table 1 . The obtained slurry mixtures were purified by silica gel flash chromatography eluting with a mixture of ethyl acetate and hexane $(1: 3)$ to obtain the pure compounds 11 in good yields.
2-(2-(Cinnamylthio)benzo[d]oxazol-5-yl)-1-(2-cyclohexenylethyl)-1H-benzo[d]imidazole-5-carboxylic Acid Methyl Ester 11\{1,1\}: ${ }^{1} \mathrm{H}$ NMR $\left(300 \mathrm{MHz}, \mathrm{CDCl}_{3}\right) \delta 8.54(\mathrm{~d}, J=$ $1.2 \mathrm{~Hz}, 1 \mathrm{H}), 8.07(\mathrm{dd}, J=8.5,1.2 \mathrm{~Hz}, 1 \mathrm{H}), 7.95(\mathrm{~d}, J=1.4 \mathrm{~Hz}$, $1 \mathrm{H}), 7.68$ (dd, $J=8.4,1.4 \mathrm{~Hz}, 1 \mathrm{H}), 7.62(\mathrm{~d}, J=8.4 \mathrm{~Hz}, 1 \mathrm{H})$, $7.48-7.30(\mathrm{~m}, 6 \mathrm{H}), 6.77(\mathrm{~d}, J=15.6 \mathrm{~Hz}, 1 \mathrm{H}), 6.44(\mathrm{~m}, 1 \mathrm{H})$, $5.20(\mathrm{~m}, 1 \mathrm{H}), 4.38(\mathrm{t}, J=7.1 \mathrm{~Hz}, 2 \mathrm{H}), 4.20(\mathrm{~d}, J=7.2 \mathrm{~Hz}, 2 \mathrm{H})$, $3.98(\mathrm{~s}, 3 \mathrm{H}), 2.37(\mathrm{t}, J=7.1 \mathrm{~Hz}, 2 \mathrm{H}), 1.85-1.83(\mathrm{~m}, 2 \mathrm{H})$, 1.74-1.70 (m, 4H), 1.48-1.44 (m, 2H); ${ }^{13} \mathrm{C}$ NMR (75 MHz, $\left.\mathrm{CDCl}_{3}\right) \delta 168.1,166.6,155.6,153.2,142.9,142.8,139.3,136.5$, $135.1,133.3,129.1,128.5,126.9,126.8,126.1,125.2,124.9$, $124.7,123.3,122.7,119.7,110.9,110.4,52.6,44.3,38.2,35.4$, 28.6, 25.5, 22.9, 22.3; MS (ESI) $m / z 550\left(\mathrm{MH}^{+}\right)$; HRMS (ESI, $m / z)$ calcd for $\mathrm{C}_{33} \mathrm{H}_{32} \mathrm{~N}_{3} \mathrm{O}_{3} \mathrm{~S}: m / z$ 550.2164; Found 550.2168; IR (KBr) 2927, 1712, 1612, 1496, 1440, $1296 \mathrm{~cm}^{-1}$.

1-Butyl-2-(2-(cinnamylthio)benzo[d]oxazol-5-yl)-1-1Hbenzo[d]imidazole-5-carboxylic Acid Methyl Ester 11\{2,1\}: ${ }^{1} \mathrm{H}$ NMR (300 MHz, $\left.\mathrm{CDCl}_{3}\right) \delta 8.55(\mathrm{~d}, J=1.4 \mathrm{~Hz}, 1 \mathrm{H}), 8.07$ (dd, $J=8.5,1.4 \mathrm{~Hz}, 1 \mathrm{H}), 7.94(\mathrm{~s}, 1 \mathrm{H}), 7.65$ (dd, $J=8.5,1.5 \mathrm{~Hz}$, $1 \mathrm{H}), 7.62$ (d, $J=8.5 \mathrm{~Hz}, 1 \mathrm{H}), 7.46$ (d, $J=8.5 \mathrm{~Hz}, 1 \mathrm{H}), 7.41-$ $7.39(\mathrm{~m}, 2 \mathrm{H}), 7.35-7.30(\mathrm{~m}, 3 \mathrm{H}), 6.77(\mathrm{~d}, J=15.6 \mathrm{~Hz}, 1 \mathrm{H})$, $6.43(\mathrm{~m}, 1 \mathrm{H}), 4.29(\mathrm{t}, J=7.2 \mathrm{~Hz}, 2 \mathrm{H}), 4.20(\mathrm{~d}, J=7.3 \mathrm{~Hz}, 2 \mathrm{H})$, 3.96 (s, 3H), 1.80 (quint, $J=7.2 \mathrm{~Hz}, 2 \mathrm{H}$ ), 1.33-1.21 (sext, $J=$ $7.2 \mathrm{~Hz}, 2 \mathrm{H}), 0.86(\mathrm{t}, J=7.2 \mathrm{~Hz}, 3 \mathrm{H}) ;{ }^{13} \mathrm{C} \mathrm{NMR}(75 \mathrm{MHz}$, $\left.\mathrm{CDCl}_{3}\right) \delta 168.0,166.6,155.5,153.2,143.1,142.8,139.3,136.6$, $135.1,129.0,128.5,127.0,126.9,126.2,125.1,124.8,123.3$, 122.7, 119.7, 110.8, 110.2, 52.5, 45.2, 35.3, 32.2, 20.3, 13.9; MS (ESI) $m / z 498\left(\mathrm{MH}^{+}\right)$; HRMS (ESI, $m / z$ ) calcd for $\mathrm{C}_{29} \mathrm{H}_{28} \mathrm{~N}_{3} \mathrm{O}_{3} \mathrm{~S} \mathrm{~m} / z$ 498.1851; Found 498.1849; IR (KBr) 2956, 1707, 1606, 1492, 1460, $1300 \mathrm{~cm}^{-1}$.

2-(2-(3,7-Dimethylocta-2,6-dienylthio)benzo[d]oxazol-5yl)-1-isobutyl-1H-benzo[d]imidazole-5-carboxylic Acid Methyl Ester 11\{3,2\}: ${ }^{1} \mathrm{H}$ NMR $\left(300 \mathrm{MHz}, \mathrm{CDCl}_{3}\right) \delta 8.53(\mathrm{~d}, J=1.3$ $\mathrm{Hz}, 1 \mathrm{H}), 8.05$ (dd, $J=8.6,1.3 \mathrm{~Hz}, 1 \mathrm{H}), 7.88$ (s, 1H), 7.62 (dd, $J=8.3,1.2 \mathrm{~Hz}, 1 \mathrm{H}), 7.58(\mathrm{~d}, J=8.3 \mathrm{~Hz}, 1 \mathrm{H}), 7.43(\mathrm{~d}, J=8.5$ $\mathrm{Hz}, 1 \mathrm{H}), 5.46(\mathrm{t}, J=7.1 \mathrm{~Hz}, 1 \mathrm{H}), 5.06(\mathrm{~m}, 1 \mathrm{H}), 4.12(\mathrm{~d}, J=7.5$ $\mathrm{Hz}, 2 \mathrm{H}), 4.02(\mathrm{~d}, J=7.6 \mathrm{~Hz}, 2 \mathrm{H}), 3.96(\mathrm{~s}, 3 \mathrm{H}), 2.13-2.04(\mathrm{~m}$, $5 \mathrm{H}), 1.79$ (s, 3H), $1.66(\mathrm{~s}, 3 \mathrm{H}), 1.59$ (s, 3H), $0.72(\mathrm{~d}, J=6.6$ $\mathrm{Hz}, 6 \mathrm{H}) ;{ }^{13} \mathrm{C} \mathrm{NMR}\left(75 \mathrm{MHz}, \mathrm{CDCl}_{3}\right) \delta 168.0,167.4,155.9$, $153.1,143.2,142.9,142.8,139.4,132.3,127.2,126.2,124.9$, 124.7, 123.9, 122.7, 119.6, 117.4, 110.8, 110.7, 52.5, 39.9, 31.0, 29.2, 26.6, 26.1, 20.4, 18.1, 16.8; MS (ESI) $m / z 518\left(\mathrm{MH}^{+}\right)$; HRMS (ESI, $m / z$ ) calcd for $\mathrm{C}_{30} \mathrm{H}_{36} \mathrm{~N}_{3} \mathrm{O}_{3} \mathrm{~S} m / z$ 518.2477; Found 518.2474; IR (KBr) 2960, 1714, 1496, 1434, 1288, 1232, $1103 \mathrm{~cm}^{-1}$.

1-Isobutyl-2-(2-(3-methylbut-2-enylthio)benzo[d]oxazol5-yl)-1H-benzo[d]imidazole-5-carboxylic Acid Methyl Ester 11\{3,3\}: ${ }^{1} \mathrm{H}$ NMR $\left(300 \mathrm{MHz}, \mathrm{CDCl}_{3}\right) \delta 8.54(\mathrm{~d}, J=1.2 \mathrm{~Hz}$, 
$1 \mathrm{H}), 8.06$ (dd, $J=8.5,1.2 \mathrm{~Hz}, 1 \mathrm{H}), 7.89$ (s, 1H), 7.64 (dd, $J=$ $8.5,1.3 \mathrm{~Hz}, 1 \mathrm{H}), 7.59$ (d, $J=8.5 \mathrm{~Hz}, 1 \mathrm{H}), 7.46(\mathrm{~d}, J=8.5 \mathrm{~Hz}$, $1 \mathrm{H}), 5.46$ (t, $J=7.6 \mathrm{~Hz}, 1 \mathrm{H}), 4.14$ (d, $J=7.5 \mathrm{~Hz}, 2 \mathrm{H}), 4.03$ (d, $J=7.8 \mathrm{~Hz}, 2 \mathrm{H}), 3.98(\mathrm{~s}, 3 \mathrm{H}), 2.10(\mathrm{~m}, 1 \mathrm{H}), 1.79(\mathrm{~d}, J=6.0 \mathrm{~Hz}$, $6 \mathrm{H}), 0.74(\mathrm{~d}, J=6.6 \mathrm{~Hz}, 6 \mathrm{H}) ;{ }^{13} \mathrm{C} \mathrm{NMR}\left(75 \mathrm{MHz}, \mathrm{CDCl}_{3}\right) \delta$ $168.1,167.3$, 155.9, 155.1, 142.9, 142.8, 139.7, 139.5, 127.2, 126.2, 124.9, 124. 122.7, 119.7, 117.6, 110.8, 110.6, 52.5, 31.1, 29.2, 26.2, 20.4, 18.4; MS (ESI) $m / z 450\left(\mathrm{MH}^{+}\right)$; HRMS (ESI, $m / z)$ calcd for $\mathrm{C}_{25} \mathrm{H}_{28} \mathrm{~N}_{3} \mathrm{O}_{3} \mathrm{~S} m / z$ 450.1851; Found 450.1853; IR (KBr) 2960, 1706, 1496, 1442, 1295, 1218, $1099 \mathrm{~cm}^{-1}$.

1-Butyl-2-(2-(3,7-dimethylocta-2,6-dienylthio)benzo[d]oxazol-5-yl)-1H-benzo[d]imidazole-5-carboxylic Acid Methyl Ester 11\{2,2\}: ${ }^{1} \mathrm{H}$ NMR (300 MHz, $\left.\mathrm{CDCl}_{3}\right) \delta 8.55$ (d, $J=1.4$ $\mathrm{Hz}, 1 \mathrm{H}), 8.07$ (dd, $J=8.5,1.4 \mathrm{~Hz}, 1 \mathrm{H}), 7.90$ (d, $J=1.1 \mathrm{~Hz}$, $1 \mathrm{H}), 7.66(\mathrm{dd}, J=8.4,1.5 \mathrm{~Hz}, 1 \mathrm{H}), 7.61(\mathrm{~d}, J=8.4 \mathrm{~Hz}, 1 \mathrm{H})$, $7.46(\mathrm{~d}, J=8.4 \mathrm{~Hz}, 1 \mathrm{H}), 5.47(\mathrm{t}, J=6.9 \mathrm{~Hz}, 1 \mathrm{H}), 5.07(\mathrm{~m}, 1 \mathrm{H})$, $4.29(\mathrm{t}, J=7.5 \mathrm{~Hz}, 2 \mathrm{H}), 4.04(\mathrm{~d}, J=7.5 \mathrm{~Hz}, 2 \mathrm{H}), 3.98(\mathrm{~s}, 3 \mathrm{H})$, 2.09-1.98 (m, 4H), $1.80(\mathrm{~s}, 3 \mathrm{H}), 1.74-1.67(\mathrm{~m}, 5 \mathrm{H}), 1.56(\mathrm{~s}$, $3 \mathrm{H}), 1.33-1.20$ (sext, $J=7.2 \mathrm{~Hz}, 2 \mathrm{H}), 0.86(\mathrm{t}, J=7.2 \mathrm{~Hz}, 3 \mathrm{H})$; ${ }^{13} \mathrm{C}$ NMR $\left(75 \mathrm{MHz}, \mathrm{CDCl}_{3}\right) \delta 168.1,167.3,155.5,153.2$, $143.2,143.0,139.3,132.3,126.9,126.1,125.0,124.8,123.9$, $122.7,119.5,117.4,110.8,110.2$, 52.5, 45.2, 39.9, 32.2, 30.1, 26.7, 26.1, 20.3, 18.1, 16.7, 13.9; MS (ESI) $m / z 518\left(\mathrm{MH}^{+}\right)$; HRMS (ESI, $m / z$ ) calcd for $\mathrm{C}_{30} \mathrm{H}_{36} \mathrm{~N}_{3} \mathrm{O}_{3} \mathrm{~S} m / z$ 518.2477; Found 518.2480; IR (KBr) 2931, 1716, 1616.

1-(2-Cyclohexenylethyl)-2-(2-(3,7-dimethylocta-2,6dienylthio)benzo[d]oxazol-5-yl)-1H-benzo[d]imidazole-5carboxylic Acid Methyl Ester 11\{1,2\}: ${ }^{1} \mathrm{H}$ NMR $(300 \mathrm{MHz}$, $\left.\mathrm{CDCl}_{3}\right) \delta 8.56(\mathrm{~s}, 1 \mathrm{H}), 8.08(\mathrm{~d}, J=8.5 \mathrm{~Hz}, 1 \mathrm{H}), 7.93(\mathrm{~s}, 1 \mathrm{H})$, $7.70(\mathrm{~d}, J=8.4 \mathrm{~Hz}, 1 \mathrm{H}), 7.61(\mathrm{~d}, J=8.4 \mathrm{~Hz}, 1 \mathrm{H}), 7.47$ (d, $J=$ $8.4 \mathrm{~Hz}, 1 \mathrm{H}), 5.46(\mathrm{t}, J=7.8 \mathrm{~Hz}, 1 \mathrm{H}), 5.19(\mathrm{~m}, 1 \mathrm{H}), 5.07(\mathrm{~m}$, $1 \mathrm{H}), 4.40(\mathrm{t}, J=7.3 \mathrm{~Hz}, 2 \mathrm{H}), 4.04(\mathrm{~d}, J=7.7 \mathrm{~Hz}, 2 \mathrm{H}), 3.98(\mathrm{~s}$, $3 \mathrm{H}), 2.37(\mathrm{t}, J=7.3 \mathrm{~Hz}, 2 \mathrm{H}), 2.09-1.96(\mathrm{~m}, 4 \mathrm{H}), 1.83-1.80$ $(\mathrm{m}, 2 \mathrm{H}), 1.79(\mathrm{~s}, 3 \mathrm{H}), 1.73-1.71(\mathrm{~m}, 4 \mathrm{H}), 1.68(\mathrm{~s}, 3 \mathrm{H}), 1.60$ (s, 3H), $1.49-1.44(\mathrm{~m}, 2 \mathrm{H}) ;{ }^{13} \mathrm{C}$ NMR (75 $\left.\mathrm{MHz}, \mathrm{CDCl}_{3}\right) \delta$ $167.9,167.5,155.8,153.3,143.2,143.0,142.9,138.9,133.2$, $132.4,126.7,126.2,125.3,125.0,123.9,122.3,119.5,117.4$, $110.9,110.6,52.6,44.4,39.9,36.1,31.2,28.6,26.7,26.1,25.5$, 22.9, 22.3, 18.1, 16.7; MS (ESI) $m / z 570\left(\mathrm{MH}^{+}\right)$; HRMS (ESI, $m / z)$ calcd for $\mathrm{C}_{34} \mathrm{H}_{40} \mathrm{~N}_{3} \mathrm{O}_{3} \mathrm{~S} m / z$ 570.2790; Found 570.2793; IR (KBr) 2970, 1710, 1608, 1502, $1440 \mathrm{~cm}^{-1}$.

1-(2-Cyclohexenylethyl)-2-(2-(3-methylbut-2-enylthio)benzo[d]oxazol-5-yl)-1H-benzo[d]imidazole-5-carboxylic Acid Methyl Ester 11\{1,3\}: ${ }^{1} \mathrm{H}$ NMR $\left(300 \mathrm{MHz}^{\mathrm{CDCl}} \mathrm{CD}_{3}\right) \delta 8.56$ (d, $J=1.4 \mathrm{~Hz}, 1 \mathrm{H}), 8.08$ (dd, $J=8.5,1.4 \mathrm{~Hz}, 1 \mathrm{H}), 7.93$ (d, $J=$ $1.5 \mathrm{~Hz}, 1 \mathrm{H}), 7.69$ (dd, $J=8.4,1.5 \mathrm{~Hz}, 1 \mathrm{H}), 7.61(\mathrm{~d}, J=8.4 \mathrm{~Hz}$, $1 \mathrm{H}), 7.48$ (d, $J=8.5 \mathrm{~Hz}, 1 \mathrm{H}), 5.47(\mathrm{t}, J=7.6 \mathrm{~Hz}, 1 \mathrm{H}), 5.19(\mathrm{~m}$, $1 \mathrm{H}), 4.39(\mathrm{t}, J=7.3 \mathrm{~Hz}, 2 \mathrm{H}), 4.03(\mathrm{~d}, J=7.8 \mathrm{~Hz}, 2 \mathrm{H}), 3.98(\mathrm{~s}$, $3 \mathrm{H}), 2.37(\mathrm{t}, J=7.3 \mathrm{~Hz}, 2 \mathrm{H}), 1.83-1.82(\mathrm{~m}, 2 \mathrm{H}), 1.79$ (d, $J=$ $6.0 \mathrm{~Hz}, 6 \mathrm{H}), 1.73-1.71(\mathrm{~m}, 2 \mathrm{H}), 1.50-1.38(\mathrm{~m}, 4 \mathrm{H}) ;{ }^{13} \mathrm{C}$ NMR $\left(75 \mathrm{MHz}, \mathrm{CDCl}_{3}\right) \delta 167.5,166.9,154.9,152.9,142.5$, $141.0,139.2,138.5,132.7,125.8,125.7,124.9,124.5,121.9$, 119.1, 117.2, 110.4, 110.0, 52.1, 43.9, 37.7, 30.7, 28.2, 25.7, 25.0, 22.5, 21.8, 17.9; MS (ESI) $m / z: 502\left(\mathrm{MH}^{+}\right)$; HRMS (ESI, $m / z$ ) calcd for $\mathrm{C}_{29} \mathrm{H}_{32} \mathrm{~N}_{3} \mathrm{O}_{3} \mathrm{~S}: m / z$ 502.2164; Found 502.2162; IR (KBr): 2927, 1712, 1602, 1492, 1442, $1303 \mathrm{~cm}^{-1}$.

2-(2-(Allylthio)benzo[d]oxazol-5-yl)-1-cyclopentyl-1Hbenzo[d]imidazole-5-carboxylic Acid Methyl Ester 11\{4,4\}: ${ }^{1} \mathrm{H} \mathrm{NMR}\left(300 \mathrm{MHz}, \mathrm{CDCl}_{3}\right) \delta 8.54(\mathrm{~d}, J=1.2 \mathrm{~Hz}, 1 \mathrm{H}), 8.01$ (dd, $J=8.5,1.2 \mathrm{~Hz}, 1 \mathrm{H}), 7.85(\mathrm{~s}, 1 \mathrm{H}), 7.62(\mathrm{~m}, 2 \mathrm{H}), 7.55$ (d, $J$ $=8.6 \mathrm{~Hz}, 1 \mathrm{H}), 6.06(\mathrm{~m}, 1 \mathrm{H}), 5.43(\mathrm{dd}, J=16.9,1.2 \mathrm{~Hz}, 1 \mathrm{H})$, $5.25(\mathrm{dd}, J=10.0,1.2 \mathrm{~Hz}, 1 \mathrm{H}), 4.94$ (quint, $J=8.6 \mathrm{~Hz}, 1 \mathrm{H}$ ), $4.00(\mathrm{~d}, J=6.5 \mathrm{~Hz}, 2 \mathrm{H}), 3.96(\mathrm{~s}, 3 \mathrm{H}), 2.37-2.30(\mathrm{~m}, 2 \mathrm{H})$,
2.17-2.05 (m, 4H), 1.77-1.72 (m, 2H); ${ }^{13} \mathrm{C}$ NMR $(75 \mathrm{MHz}$, $\left.\mathrm{CDCl}_{3}\right) \delta 167.5,166.1,155.5,152.8,143.1,142.2,136.4,131.8$, $126.6,125.9,124.4,123.8,122.5,119.6,119.4,111.5,110.4$, 57.8, 52.1, 34.9, 30.4, 25.2; MS (ESI) $m / z 434\left(\mathrm{MH}^{+}\right)$; HRMS (ESI, $m / z$ ) calcd for $\mathrm{C}_{24} \mathrm{H}_{24} \mathrm{~N}_{3} \mathrm{O}_{3} \mathrm{~S} \mathrm{~m} / z$ 434.1538; Found 434.1535; IR (KBr) 2952, 1714, 1614, 1498, 1436, 1298, 1222 $\mathrm{cm}^{-1}$.

1-Cyclopentyl-2-(2-(3,7-dimethylocta-2,6-dienylthio)benzo[d]oxazol-5-yl)-1 H-benzo[d]imidazole-5-carboxylic Acid Methyl Ester 11\{4,2\}: ${ }^{1} \mathrm{H}$ NMR (300 MHz, $\left.\mathrm{CDCl}_{3}\right) \delta 8.53$ $(\mathrm{d}, J=1.2 \mathrm{~Hz}, 1 \mathrm{H}), 7.99$ (dd, $J=8.5,1.2 \mathrm{~Hz}, 1 \mathrm{H}), 7.84(\mathrm{~s}, 1 \mathrm{H})$, $7.63-7.59(\mathrm{~m}, 2 \mathrm{H}), 7.54(\mathrm{~d}, J=8.6 \mathrm{~Hz}, 1 \mathrm{H}), 5.44(\mathrm{t}, J=7.6$ $\mathrm{Hz}, 1 \mathrm{H}), 5.05(\mathrm{~m}, 1 \mathrm{H}), 4.91(\mathrm{q}, J=8.1 \mathrm{~Hz}, 1 \mathrm{H}), 4.02(\mathrm{~d}, J=$ $7.5 \mathrm{~Hz}, 2 \mathrm{H}), 3.95$ (s, 3H), 2.38-2.26 (m, 2H), 2.16-1.93 (m, $10 \mathrm{H}), 1.78(\mathrm{~s}, 3 \mathrm{H}), 1.76-1.72(\mathrm{~m}, 2 \mathrm{H}), 1.65(\mathrm{~s}, 3 \mathrm{H}), 1.59(\mathrm{~s}$, $3 \mathrm{H}) ;{ }^{13} \mathrm{C} \mathrm{NMR}\left(75 \mathrm{MHz}, \mathrm{CDCl}_{3}\right) \delta 167.9,167.3,156.0,153.1$, $143.6,143.1,142.8,136.8,132.3,126.2,125.5,124.2,123.7$, $123.9,122.9,119.6,117.4,111.9,110.8,58.2,52.5,37.5,31.0$, 30.8, 26.6, 26.0, 25.6, 18.1, 16.7; MS (ESI) $m / z 530\left(\mathrm{MH}^{+}\right)$; HRMS (ESI, $m / z$ ) calcd for $\mathrm{C}_{31} \mathrm{H}_{36} \mathrm{~N}_{3} \mathrm{O}_{3} \mathrm{~S} m / z$ 530.2477, Found 530.2480; IR (KBr) 2956, 1714, 1614, 1496, 1441, $1298,1220 \mathrm{~cm}^{-1}$.

2-(2-(Benzylthio)benzo[d]oxazol-5-yl)-1-(thiophen-2-ylmethyl)-1H-benzo[d]imidazole-5-carboxylic Acid Methyl Ester 11\{5,5\}: ${ }^{1} \mathrm{H}$ NMR $\left(300 \mathrm{MHz}, \mathrm{CDCl}_{3}\right) \delta 8.57$ (d, $J=$ $1.3 \mathrm{~Hz}, 1 \mathrm{H}$ ), 8.05 (dd, $J=8.6,1.3 \mathrm{~Hz}, 1 \mathrm{H}), 7.99$ (d, $J=1.5 \mathrm{~Hz}$, $1 \mathrm{H}), 7.68$ (dd, $J=8.4,1.5 \mathrm{~Hz}, 1 \mathrm{H}), 7.59$ (d, $J=8.4 \mathrm{~Hz}, 1 \mathrm{H})$, $7.50-7.47$ (m, 2H), $7.40(\mathrm{~d}, J=5.5 \mathrm{~Hz}, 2 \mathrm{H}), 7.37-7.31(\mathrm{~m}$, $2 \mathrm{H}), 7.24(\mathrm{~d}, J=5.5 \mathrm{~Hz}, 1 \mathrm{H}), 6.95(\mathrm{dd}, J=5.0,3.5 \mathrm{~Hz}, 1 \mathrm{H})$, $6.85(\mathrm{~d}, J=3.5 \mathrm{~Hz}, 1 \mathrm{H}), 5.62(\mathrm{~s}, 2 \mathrm{H}), 4.60(\mathrm{~s}, 2 \mathrm{H}), 3.98(\mathrm{~s}$, $3 \mathrm{H}) ;{ }^{13} \mathrm{C} \mathrm{NMR}\left(75 \mathrm{MHz}, \mathrm{CDCl}_{3}\right) \delta 167.9,166.8,155.4,153.4$, $142.9,142.8,139.2,138.6,135.9,129.5,129.2,128.5,127.7$, $126.4,126.3,126.2,126.1,125.6,125.2,122.7,119.9,110.9$, 110.6, 52.6, 44.7, 37.1; MS (ESI) $m / z 512\left(\mathrm{MH}^{+}\right)$; HRMS (ESI, $m / z$ ) calcd for $\mathrm{C}_{28} \mathrm{H}_{22} \mathrm{~N}_{3} \mathrm{O}_{3} \mathrm{~S}_{2} \mathrm{~m} / z$ 512.1103, Found 512.1104; IR (KBr) 3052, 1714, 1616, 1495, 1433, 1300, 1216 $\mathrm{cm}^{-1}$.

2-(2-(Allylthio)benzo[d]oxazol-5-yl)-1-(thiophen-2-ylmethyl)-1H-benzo[d]imidazole-5-carboxylic Acid Methyl Ester 11\{5,4\}: ${ }^{1} \mathrm{H}$ NMR (300 MHz, $\left.\mathrm{CDCl}_{3}\right) \delta 8.56(\mathrm{~d}, J=1.3 \mathrm{~Hz}$, $1 \mathrm{H}), 8.05$ (dd, $J=8.4,1.3 \mathrm{~Hz}, 1 \mathrm{H}), 7.96(\mathrm{~d}, J=1.5 \mathrm{~Hz}, 1 \mathrm{H})$, 7.69 (dd, $J=8.4,1.5 \mathrm{~Hz}, 1 \mathrm{H}), 7.58(\mathrm{~d}, J=8.4 \mathrm{~Hz}, 1 \mathrm{H}), 7.42$ (d, $J=8.5 \mathrm{~Hz}, 1 \mathrm{H}), 7.24(\mathrm{~d}, J=5.1 \mathrm{~Hz}, 1 \mathrm{H}), 6.95(\mathrm{dd}, J=5.0,3.4$ $\mathrm{Hz}, 1 \mathrm{H}), 6.84(\mathrm{~d}, J=3.4 \mathrm{~Hz}, 1 \mathrm{H}), 6.05(\mathrm{~m}, 1 \mathrm{H}), 5.62(\mathrm{~s}, 2 \mathrm{H})$, $5.44(\mathrm{dd}, J=16.9,1.1 \mathrm{~Hz}, 1 \mathrm{H}), 5.25(\mathrm{dd}, J=10.1,1.1 \mathrm{~Hz}, 1 \mathrm{H})$, 4.00 (d, $J=6.5 \mathrm{~Hz}, 2 \mathrm{H}), 3.97(\mathrm{~s}, 3 \mathrm{H}) ;{ }^{13} \mathrm{C}$ NMR $(75 \mathrm{MHz}$, $\mathrm{CDCl}_{3}$ ) $\delta 167.9,166.6,155.4,153.4,142.9,142.8,139.2,138.6$, $132.2,127.7,126.3,126.1,125.5,125.1,124.5,120.5,120.4$, 120.0, 119.9, 110.9, 110.6, 52.6, 44.7, 35.4; MS (ESI) $\mathrm{m} / z 462$ $\left(\mathrm{MH}^{+}\right)$; HRMS (ESI, $m / z$ ) calcd for $\mathrm{C}_{24} \mathrm{H}_{20} \mathrm{~N}_{3} \mathrm{O}_{3} \mathrm{~S}_{2} \mathrm{~m} / z$ 462.0946; Found 462.0949; IR (KBr) 2929, 1714, 1614, 1498, $1434,1296 \mathrm{~cm}^{-1}$.

2-(2-(3,7-Dimethylocta-2,6-dienylthio)benzo[d]oxazol-5yl)-1-(thiophen-2-ylmethyl)-1H-benzo[d] imidazole-5-carboxylic Acid Methyl Ester $11\{5,2\}:{ }^{1} \mathrm{H}$ NMR $\left(300 \mathrm{MHz}, \mathrm{CDCl}_{3}\right) \delta$ 8.57 (d, $J=1.4 \mathrm{~Hz}, 1 \mathrm{H}), 8.05$ (dd, $J=7.2,1.4 \mathrm{~Hz}, 1 \mathrm{H}), 7.95$ (d, $J=1.5 \mathrm{~Hz}, 1 \mathrm{H}), 7.69$ (dd, $J=8.4,1.5 \mathrm{~Hz}, 1 \mathrm{H}), 7.58(\mathrm{~d}, J=8.4$ $\mathrm{Hz}, 1 \mathrm{H}), 7.42$ (d, $J=8.5 \mathrm{~Hz}, 1 \mathrm{H}), 7.24(\mathrm{~d}, J=5.0 \mathrm{~Hz}, 1 \mathrm{H})$, 6.95 (dd, $J=5.0,3.4 \mathrm{~Hz}, 1 \mathrm{H}), 6.84(\mathrm{~d}, J=3.4 \mathrm{~Hz}, 1 \mathrm{H}), 5.62$ (s, $2 \mathrm{H}), 5.40(\mathrm{~d}, J=7.1 \mathrm{~Hz}, 2 \mathrm{H}), 5.08(\mathrm{~m}, 1 \mathrm{H}), 4.03(\mathrm{~d}, J=7.6$ $\mathrm{Hz}, 2 \mathrm{H}), 3.91$ (s, 3H), 2.10-2.05 (m, 3H), 1.79 (s, 3H), 1.71 (s, 3H), $1.61(\mathrm{~s}, 3 \mathrm{H}) ;{ }^{13} \mathrm{C} \mathrm{NMR}\left(75 \mathrm{MHz}, \mathrm{CDCl}_{3}\right) \delta 167.8$, $167.5,155.4,153.5,143.2,142.9,142.0,139.1,138.5,132.3$, 
$127.7,126.3,126.2,126.1,126.0,125.9,123.9,122.8,119.7$, 117.4, 110.9, 110.6, 52.6, 44.7, 39.9, 31.0, 26.1, 25.9, 18.1, 16.8; MS (ESI) $m / z 558\left(\mathrm{MH}^{+}\right)$; HRMS (ESI, $\left.m / z\right)$ calcd for $\mathrm{C}_{31} \mathrm{H}_{32} \mathrm{~N}_{3} \mathrm{O}_{3} \mathrm{~S}_{2} \mathrm{~m} / z$ 558.1885; Found 558.1887; IR ( $\mathrm{KBr}$ ): 2929, 1710, 1610, 1496, 1439, 1296, $1219 \mathrm{~cm}^{-1}$.

2-(2-(Allylthio)benzo[d]oxazol-5-yl)-1-(furan-2-ylmethyl)$1 \mathrm{H}$-benzo[d]imidazole-5-Carboxylic Acid Methyl Ester 11\{6,4\}: ${ }^{1} \mathrm{H}$ NMR (300 MHz, $\left.\mathrm{CDCl}_{3}\right) \delta 8.56$ (d, $J=1.3 \mathrm{~Hz}$, $1 \mathrm{H}), 8.08$ (d, $J=1.9 \mathrm{~Hz}, 1 \mathrm{H}), 8.06(\mathrm{dd}, J=8.4,1.3 \mathrm{~Hz}, 1 \mathrm{H})$, $7.78(\mathrm{dd}, J=8.4,1.7 \mathrm{~Hz}, 1 \mathrm{H}), 7.62(\mathrm{~d}, J=8.4 \mathrm{~Hz}, 1 \mathrm{H}), 7.52(\mathrm{~d}$, $J=8.5 \mathrm{~Hz}, 1 \mathrm{H}), 7.42(\mathrm{dd}, J=2.5,1.9 \mathrm{~Hz}, 1 \mathrm{H}), 6.38(\mathrm{dd}, J=$ $3.2,1.9 \mathrm{~Hz}, 1 \mathrm{H}), 6.31(\mathrm{~d}, J=3.2 \mathrm{~Hz}, 1 \mathrm{H}), 6.09(\mathrm{~m}, 1 \mathrm{H})$, 5.44(dd, $J=16.9,1.1 \mathrm{~Hz}, 1 \mathrm{H}), 5.40$ (s, 2H), 5.26 (dd, $J=10.1$, $1.1 \mathrm{~Hz}, 1 \mathrm{H}), 4.00(\mathrm{~d}, J=6.5 \mathrm{~Hz}, 2 \mathrm{H}), 3.97(\mathrm{~s}, 3 \mathrm{H}) ;{ }^{13} \mathrm{C} \mathrm{NMR}$ $\left(75 \mathrm{MHz}, \mathrm{CDCl}_{3}\right) \delta 167.8,166.7,155.5,153.5,148.8,145.5$, $145.0,143.7,142.8,139.2,133.2,132.2,126.6,125.7,125.4$, $122.4,120.2,120.0,111.1,110.9,110.8,52.6,42.8,33.6$; MS (ESI) $m / z 446\left(\mathrm{MH}^{+}\right)$; HRMS (ESI, $\left.m / z\right)$ calcd for $\mathrm{C}_{24} \mathrm{H}_{20} \mathrm{~N}_{3} \mathrm{O}_{4} \mathrm{~S} m / z$ 446.1174; Found 446.1175; IR (KBr) $2925,1710,1614,1500,1452,1288 \mathrm{~cm}^{-1}$.

2-(2-(3,7-Dimethylocta-2,6-dienylthio)benzo[d]oxazol-5yl)-1-(furan-2-ylmethyl)-1H-benzo[d]imidazole-5-carboxylic Acid Methyl Ester 11\{6,2\}: ${ }^{1} \mathrm{H}$ NMR (300 MHz, $\left.\mathrm{CDCl}_{3}\right) \delta 8.57$ (s, $1 \mathrm{H}), 8.06(\mathrm{t}, J=8.3 \mathrm{~Hz}, 2 \mathrm{H}), 7.77(\mathrm{~d}, J=8.5 \mathrm{~Hz}, 1 \mathrm{H}), 7.62$ $(\mathrm{d}, J=8.5 \mathrm{~Hz}, 1 \mathrm{H}), 7.52(\mathrm{~d}, J=8.5 \mathrm{~Hz}, 1 \mathrm{H}), 7.42(\mathrm{~s}, 1 \mathrm{H}), 6.38$ (s, 1H), $6.31(\mathrm{~d}, J=3.1 \mathrm{~Hz}, 1 \mathrm{H}), 5.46(\mathrm{t}, J=7.1 \mathrm{~Hz}, 1 \mathrm{H}), 5.39$ $(\mathrm{s}, 2 \mathrm{H}), 5.07(\mathrm{~m}, 1 \mathrm{H}), 4.06(\mathrm{~d}, J=7.6 \mathrm{~Hz}, 2 \mathrm{H}), 3.93(\mathrm{~s}, 3 \mathrm{H})$, 2.09-2.06 (m, 4H), $1.80(\mathrm{~s}, 3 \mathrm{H}), 1.66(\mathrm{~s}, 3 \mathrm{H}), 1.61(\mathrm{~s}, 3 \mathrm{H})$; ${ }^{13} \mathrm{C}$ NMR $\left(75 \mathrm{MHz}, \mathrm{CDCl}_{3}\right) \delta 167.8,167.4,155.5,153.5$, $148.9,143.7,143.2,142.9,142.0,139.2,132.3,126.5,126.3$, $123.9,122.8,119.7,117.4,111.1,110.9,110.6,109.5,52.6,42.8$, 39.9, 30.1, 26.7, 26.1, 25.9, 18.1, 16.7; MS (ESI) $\mathrm{m} / z 542$ $\left(\mathrm{MH}^{+}\right)$; HRMS (ESI, $m / z$ ) calcd for $\mathrm{C}_{31} \mathrm{H}_{32} \mathrm{~N}_{3} \mathrm{O}_{4} \mathrm{~S}_{2} \mathrm{~m} / z$ 542.2113; Found 542.2110; IR (KBr) 2925, 1716, 1616, 1448, $1295,1224 \mathrm{~cm}^{-1}$.

2-(2-(Allylthio)benzo[d]oxazol-5-yl)-1-butyl-1H-benzo[d]imidazole-5-carboxylic Acid Methyl Ester 11\{2,4\}: ${ }^{1} \mathrm{H}$ NMR $\left(300 \mathrm{MHz}, \mathrm{CDCl}_{3}\right) \delta 8.52(\mathrm{~d}, J=1.4 \mathrm{~Hz}, 1 \mathrm{H}), 8.04(\mathrm{dd}, J=$ 8.5, $1.4 \mathrm{~Hz}, 1 \mathrm{H}), 7.88$ (d, $J=1.5 \mathrm{~Hz}, 1 \mathrm{H}), 7.64$ (dd, $J=8.3,1.5$ $\mathrm{Hz}, 1 \mathrm{H}), 7.58(\mathrm{~d}, J=8.3 \mathrm{~Hz}, 1 \mathrm{H}), 7.44(\mathrm{~d}, J=8.5 \mathrm{~Hz}, 1 \mathrm{H})$, $6.04(\mathrm{~m}, 1 \mathrm{H}), 5.42(\mathrm{dd}, J=16.8,1.2 \mathrm{~Hz}, 1 \mathrm{H}), 5.24(\mathrm{dd}, J=$ $10.2,1.2 \mathrm{~Hz}, 1 \mathrm{H}), 4.26(\mathrm{t}, J=7.5 \mathrm{~Hz}, 2 \mathrm{H}), 3.98(\mathrm{~d}, J=6.9 \mathrm{~Hz}$, $2 \mathrm{H}$ ), $3.96(\mathrm{~s}, 3 \mathrm{H}$ ), 1.77 (quint, $J=7.5 \mathrm{~Hz}, 2 \mathrm{H}$ ), 1.28-1.20 (sext, $J=7.5 \mathrm{~Hz}, 2 \mathrm{H}), 0.83(\mathrm{t}, J=7.5 \mathrm{~Hz}, 3 \mathrm{H}) ;{ }^{13} \mathrm{C}$ NMR $(75$ $\left.\mathrm{MHz} \mathrm{CDCl}_{3}\right) \delta 168.0,166.6,155.5,153.2,143.0,142.7,139.2$, $132.2,126.9,126.2,125.0,124.8,122.7,120.0,119.6,110.8$, $110.3,52.5,45.2,35.4,32.2,20.3,13.9 ; \mathrm{MS}$ (ESI) $\mathrm{m} / z 422$ $\left(\mathrm{MH}^{+}\right.$); HRMS (ESI, $m / z$ ) calcd for $\mathrm{C}_{23} \mathrm{H}_{24} \mathrm{~N}_{3} \mathrm{O}_{3} \mathrm{~S} m / z$ 422.1538; Found 422.1539; IR (KBr) 2960, 2933, 1704, 1680, 1496, 1442, 1278, $1220 \mathrm{~cm}^{-1}$.

2-(2-(Benzylthio)benzo[d]oxazol-5-yl)-1-butyl-1H-benzo[d]imidazole-5-carboxylic Acid Methyl Ester 11\{2,5\}: ${ }^{1} \mathrm{H}$ NMR (300 MHz, $\left.\mathrm{CDCl}_{3}\right) \delta 8.55(\mathrm{~d}, J=1.4 \mathrm{~Hz}, 1 \mathrm{H}), 8.08$ $(\mathrm{dd}, J=8.5,1.4 \mathrm{~Hz}, 1 \mathrm{H}), 7.94(\mathrm{~d}, J=1.5 \mathrm{~Hz}, 1 \mathrm{H}), 7.66(\mathrm{dd}, J=$ 8.4, $1.5 \mathrm{~Hz}, 1 \mathrm{H}), 7.60(\mathrm{~d}, J=8.4 \mathrm{~Hz}, 1 \mathrm{H}), 7.51-7.45(\mathrm{~m}, 3 \mathrm{H})$, $7.40-7.32(\mathrm{~m}, 3 \mathrm{H}), 4.65(\mathrm{~s}, 2 \mathrm{H}), 4.29(\mathrm{t}, J=7.3 \mathrm{~Hz}, 2 \mathrm{H}), 3.98$ (s, $3 \mathrm{H}$ ), 1.82 (quint, $J=7.3 \mathrm{~Hz}, 2 \mathrm{H}$ ), 1.27 (sext, $J=7.3 \mathrm{~Hz}$, $2 \mathrm{H}), 0.87$ (t, $J=7.3 \mathrm{~Hz}, 3 \mathrm{H}) ;{ }^{13} \mathrm{C} \mathrm{NMR}\left(75 \mathrm{MHz} \mathrm{CDCl}_{3}\right) \delta$ $167.7,167.0,154.8,153.5,142.8,138.6,135.9,129.6,129.5$, $129.2,128.5,126.4,125.8,125.3,122.1,122.0,119.7,111.1$, 110.6, 52.6, 45.4, 37.1, 32.1, 20.3, 13.9; MS (ESI) $\mathrm{m} / z 472$ $\left(\mathrm{MH}^{+}\right) ; \mathrm{HRMS}$ (ESI, $m / z$ ) calcd for $\mathrm{C}_{27} \mathrm{H}_{26} \mathrm{~N}_{3} \mathrm{O}_{3} \mathrm{~S} m / z$
472.1695; Found 472.1696; IR (KBr) 2861, 1710, 1612, 1498, $1442,1288,1218 \mathrm{~cm}^{-1}$.

1-Butyl-2-(2-thioxo-2,3-dihydro-1,3-benzoxazol-5-yl)-1Hbenzo[d]imidazole-5-carboxylic Acid Methyl Ester $(\boldsymbol{A}):{ }^{1} \mathrm{H}$ $\operatorname{NMR}\left(600 \mathrm{MHz}, \mathrm{CDCl}_{3}\right) \delta 8.66(\mathrm{~d}, J=1.5 \mathrm{~Hz}, 1 \mathrm{H}), 8.07$ (dd, $J=8.6,1.5 \mathrm{~Hz}, 1 \mathrm{H}), 7.72(\mathrm{~d}, J=1.4 \mathrm{~Hz}, 1 \mathrm{H}), 7.46(\mathrm{~d}, J=8.6$ $\mathrm{Hz}, 1 \mathrm{H}), 7.44$ (d, $J=1.4 \mathrm{~Hz}, 1 \mathrm{H}), 7.41(\mathrm{~d}, J=8.4 \mathrm{~Hz}, 1 \mathrm{H})$, $4.30(\mathrm{t}, J=7.6 \mathrm{~Hz}, 2 \mathrm{H}$ ), $3.94(\mathrm{~s}, 3 \mathrm{H}), 1.79$ (quint, $J=7.6 \mathrm{~Hz}$, $2 \mathrm{H}), 1.26$ (sext, $J=7.6 \mathrm{~Hz}, 3 \mathrm{H}), 0.87(\mathrm{t}, J=7.6 \mathrm{~Hz}, 3 \mathrm{H}) ;{ }^{13} \mathrm{C}$ NMR $\left(150 \mathrm{MHz}, \mathrm{CDCl}_{3}\right) \delta 181.9,167.4,154.3,150.1,141.6$, $138.4,132.7,126.5,125.0,123.9,122.1,111.6,110.3,110.2$, 52.2, 44.9, 31.8, 19.9, 13.5; MS (EI) $m / z$ 381 (M+ ${ }^{+}$); HRMS (EI, $m / z)$ calcd for $\mathrm{C}_{20} \mathrm{H}_{19} \mathrm{~N}_{3} \mathrm{O}_{3} \mathrm{~S}_{1} m / z$ 381.1147; Found 381.1149.

\section{ASSOCIATED CONTENT}

\section{S Supporting Information}

General experimental procedures and representative ${ }^{1} \mathrm{H}$ NMR, ${ }^{13} \mathrm{C}$ NMR, crude HPLC, LRMS, HRMS, and FT-IR spectral data of compounds 11, as well as the intermediates. This material is available free of charge via the Internet at http:// pubs.acs.org.

\section{AUTHOR INFORMATION}

\section{Corresponding Author}

*E-mail: cmsun@mail.nctu.edu.tw.

\section{Notes}

The authors declare no competing financial interest.

\section{ACKNOWLEDGMENTS}

The authors thank the National Science Council of Taiwan for the financial assistance and the authorities of the National Chiao Tung University for providing the laboratory facilities. This paper is particularly supported by "Aim for the Top University Plan” of the National Chiao Tung University and Ministry of Education, Taiwan, R.O.C.

\section{REFERENCES}

(1) (a) Schreiber, S. L. Target-oriented and diversity-oriented organic synthesis in drug discovery. Science 2000, 287, 1964-1969. (b) Stockwell, B. R. Exploring biology with small organic molecules. Nature 2004, 432, 846-854. (c) Tan, D. S. Diversity-oriented synthesis: exploring the intersections between chemistry and biology. Nat. Chem. Biol. 2005, 1, 74-84.

(2) Breinbauer, R; Vetter, I. R.; Waldman, H. From protein domains to drug candidates-Natural products as guiding principles in the design and synthesis of compound libraries. Angew. Chem.,Int. Ed. 2002, 41, 2878-2890.

(3) (a) Wessjohann, L. A. Synthesis of natural-product-based compound libraries. Curr. Opin. Chem. Bio. 2000, 4, 303-309. (b) Wipf, P. Synthetic aspects of combinatorial chemistry. Pharm. News 2002, 9, 157-169.

(4) (a) Merrifield, R. B. Solid phase synthesis. Science 1986, 232, 341-347. (b) Plante, O. J.; Palmacci, E. R.; Seeberger, P. H. Automated solid-phase synthesis of oligosaccharides. Science 2001, 291, 1523-1527. (c) Krachňák, V.; Holladay, M. W. Solid phase heterocyclic chemistry. Chem. Rev. 2002, 102, 61-91. (d) Nielsen, T. E.; Meldal, M. Solid-phase synthesis of complex and pharmacologically interesting heterocycles. Curr. Opin. Drug Discovery Dev. 2009, 12, 798-810. (e) Lu, J.; Toy, P. H. Organic polymer supports for synthesis and for reagent and catalyst immobilization. Chem. Rev. 2009, 109, 815-838. (f) Chen, R.; Tolbert, T. J. Study of on-resin convergent synthesis of $\mathrm{N}$-linked glycopeptides containing a large high mannose N-linked oligosaccharide. J. Am. Chem. Soc. 2010, 132, $3211-3216$ 
(5) (a) Miao, W.; Chan, T. H. Exploration of ionic liquids as soluble supports for organic synthesis. Demonstration with a Suzuki coupling reaction. Org. Lett. 2003, 5, 5003-5006. (b) Fraga-Dubreuil, J.; Bazureau, J. P. Efficient combination of task-specific ionic liquid and microwave dielectric heating applied to one-pot three component synthesis of a small library of 4-thiazolidinones. Tetrahedron 2003, 59, 6121-6130. (c) He, X.; Chan, T. H. Ionic-tag-assisted oligosaccharide synthesis. Synthesis 2006, 1645-1648. (d) Donga, R. A.; Khaliq-UzZaman, S. M.; Chan, T. H.; Damha, M. J. A novel approach to oligonucleotide synthesis using an imidazolium ion tag as a soluble support. J. Org. Chem. 2006, 71, 7907-7910.

(6) (a) Wasserschied, P., Welton, T. Ionic Liquids in Synthesis; WileyVCH Verlag: Stuttgart, Germany, 2002. (b) Zhao, G.; Jiang, T.; Gao, H.; Han, B.; Huang, J.; Sun, D. Mannich reaction using acidic ionic liquids as catalysts and solvents. Green Chem. 2004, 6, 75-77. (c) Ramon, D. J.; Yus, M. Asymmetric multicomponent reactions (AMCRs): The new frontier. Angew. Chem., Int. Ed. 2005, 44, 16021634. (d) Flanagan, S.; Hall, E.; Bowie, W.; Fuhs, J. W.; Logan, R.; Maniei, F.; Hunt, A. A design-of-experiments approach to modeling activity coefficients in solvent mixtures: a case study using platinum(II) acetylacetonate in mixtures of acetone, cyclohexanol, 1,2,3,4tetrahydronaphthalene and propylene carbonate. Green Chem. 2005, 7, 333-338. (e) Toma, Š.; Mečiarová, M.; Šebesta, R. Are ionic liquids suitable media for organocatalytic reactions? Eur. J. Org. Chem. 2009, 321-327. (f) Huo, C.; Chan, T. H. A novel liquid-phase strategy for organic synthesis using organic ions as soluble supports. Chem. Soc. Rev. 2010, 39, 2977-3006. (g) Isambert, N.; del Mar S, D, M.; Plaquevent, J. C.; Génisson, Y.; Rodriguez, J.; Constantieu, T. Multicomponent reactions and ionic liquids: a perfect synergy for eco-compatible heterocyclic synthesis. Chem. Soc. Rev. 2011, 40, $1347-1357$.

(7) (a) Miao, W.; Chan, T. H. Ionic-liquid-supported peptide synthesis demonstrated by the synthesis of $\mathrm{Leu}^{5}$-enkephalin. J. Org. Chem. 2005, 70, 3251-3255. (b) Miao, W.; Chan, T. H. Ionic-liquidsupported synthesis: A novel liquid-phase strategy for organic synthesis. Acc. Chem. Res. 2006, 39, 897-908. (c) He, X.; Chan, T. $\mathrm{H}$. Structurally defined imidazolium-type ionic oligomers as soluble/ solid support for peptide synthesis. Org. Lett. 2007, 9, 2681-2684. (d) Martins, M. A. P.; Frizzo, C. P.; Moreira, D. N.; Zanatta, N.; Bonacorso, H. G. Ionic liquids in heterocyclic synthesis. Chem. Rev. 2008, 108, 2015-2050.

(8) (a) Zambrowicz, B. P.; Sands, A. T. Knockouts model the 100 best-selling drugs-Will they model the next 100? Nat. Rev. Drug Discovery 2003, 2, 38-51. (b) King, A. R. Gluten content of the top 200 medications of 2008: A follow-up to the impact of celiac sprue on patients' medication choices. Hosp. Pharm. 2009, 44, 984-99.

(9) (a) Yellol, G. S.; Tsai, W. C.; Sun, C. M. Novel cyclization of bisBoc-guanidines: expeditive traceless synthesis of 1,3,5-oxadiazinones under microwave condition. Chem. Commun. 2010, 46, 9170-9172. (b) Chen, C. H.; Kuo, J.; Yellol, G. S.; Sun, C. M. Microwave-assisted tandem transformation on an ionic-liquid support: Efficient synthesis of pyrrolo/pyridobenzimidazolones and isoindolinone-fused benzimidazoles. Chem. Asian J. 2011, 6, 2471-2480. (c) Thummanagoti, S.; Yellol, G. S.; Sun, C. M. Regioselective, unconventional PictetSpengler cyclization strategy toward the synthesis of benzimidazolelinked imidazoquinoxalines on a soluble polymer support. Chem. Asian J. 2011, 6, 1557-1565. (d) Chen, C. H.; Yellol, G. S.; Lin, P. T.; Sun, C. M. Base-catalyzed Povarov reaction: An unusual $[1,3]$ sigmatropic rearrangement to dihydropyrimidobenzimidazoles. Org. Lett. 2011, 13, $5120-5123$

(10) (a) Hirashima, S.; Suzuki, T.; Ishida, T.; Noji, S.; Ando, I.; Komatsu, M.; Ikede, S.; Hashimoto, H. Benzimidazole derivatives bearing substituted biphenyls as hepatitis C virus NS5B RNAdependent RNA polymerase inhibitors: Structure-activity relationship studies and identification of a potent and highly selective inhibitor JTK-109. J. Med. Chem. 2006, 49, 4721-4736. (b) Li, Y. F.; Wang, G. F.; He, P. L.; Huang, W. G.; Gao, H. Y.; Tang, W.; Luo, Y.; Feng, C. L.; Shi, L. P.; Ren, Y. D.; Lu, W.; Zuo, J. P. Synthesis and AntiHepatitis B Virus Activity of Novel Benzimidazole Derivatives. J. Med.
Chem. 2006, 49, 4790-4794. (c) Hasegawa, M.; Nishigaki, N.; Washio, Y.; Kano, K.; Harris, P. A.; Sato, H.; Mori, I.; West, R. I.; Shibahara, M.; Toyoda, H.; Wang, L.; Nolte, R. T.; Veal, J. M.; Cheung, M. Discovery of Novel Benzimidazoles as Potent Inhibitors of TIE-2 and VEGFR-2 Tyrosine Kinase Receptors. J. Med. Chem. 2007, 50, 4453-4470.

(11) (a) Paramashivappa, R.; Kumar, P. P.; Rao, P. V. S.; Rao, A. S. Design, synthesis, and biological evaluation of benzimidazole/ benzothiazole and benzoxazole derivatives as cyclooxygenase inhibitors. Biorg. Med. Chem. Lett. 2003, 13, 657-660. (b) Siracusa, M. A.; Salerno, L.; Modica, M. N.; Pittala, V.; Romeo, G.; Amato, M. E.; Nowak, M.; Bojarski, A. J.; Mereghetti, I.; Cagnotto, A.; Mennini, T. Synthesis of new arylpiperazinylalkylthiobenzimidazole, benzothiazole, or benzoxazole derivatives as potent and selective 5-HT1A serotonin receptor ligands. J. Med. Chem. 2008, 51, 4529-4538-4529. (c) Harmange, J. C.; Weiss, M. M.; Germain, J.; Polverino, A. J.; Borg, G.; Bready, J.; Chen, D.; Choquette, D.; Coxon, A.; DeMelfi, T.; DiPietro, L.; Doerr, N.; Estrada, J.; Flynn, J.; Graceffa, R. F.; Harriman, S. P.; Kaufman, S.; La, D. S.; Long, A.; Martin, M. W.; Neervannan, S.; Patel, V. F.; Potashman, M.; Regal, K.; Roveto, P. M.; Schrag, M. L.; Starnes, C.; Tasker, A.; Teffera, Y.; Wang, L.; White, R. D.; Whittington, D. A.; Zanon, R. Naphthamides as novel and potent vascular endothelial growth factor receptor tyrosine kinase inhibitors: Design, synthesis, and evaluation. J. Med. Chem. 2008, 51, 1649-1667. (12) (a) Wang, F.; Heuske, J. R. Solid-phase synthesis of benzoxazoles via Mitsunobu reaction. Tetrahedron Lett. 1997, 38, 6529-6532. (b) Pottorf, R. S.; Chadha, N. K.; Katkevics, M.; Ozola, V.; Suna, E.; Ghane, H.; Regberg, T.; Player, M. R. Parallel synthesis of benzoxazoles via microwave-assisted dielectric heating. Tetrahedron Lett. 2003, 44, 175-178. (c) Evindar, G.; Batey, R. A. Parallel synthesis of a library of benzoxazoles and benzothiazoles using ligandaccelerated copper-catalyzed cyclizations of ortho-halobenzanilides. J. Org. Chem. 2006, 71, 1802-1808.

(13) Chanda, K.; Maiti, B.; Chung, W. S.; Sun, C. M. Novel approach towards 2-substituted aminobenzimidazoles on imidazolium ion tag under focused microwave irradiation. Tetrahedron 2011, 67, 62146220 .

(14) (a) Andrzejewska, M.; Yepez-Mulia, L.; Tapia, A.; CedilloRivera, R.; Laudy, A. E.; Staršciak, B. J.; Kazimierczuk, Z. Synthesis and antiprotozoal and antibacterial activities of S-substituted 4,6-dibromoand 4,6-dichloro-2-mercaptobenzimidazoles. Eur. J. Pharm. Sci. 2004, 21, 323-329. (b) Hwu, J. R.; Singha, R.; Hong, S. C.; Chang, Y. H.; Das, A. R.; Vliegen, I.; DeClercq, E.; Neyts, J. Synthesis of new benzimidazole-coumarin conjugates as anti-hepatitis $\mathrm{C}$ virus agents. Antiviral Res. 2007, 77, 157-162.

(15) Final product $11 \mathrm{~b}$ was crystallized by slow evaporation of a solution of ethyl acetate-hexane $(1: 1, \mathrm{v} / \mathrm{v})$ at room temperature. Crystal data: $\mathrm{C}_{29} \mathrm{H}_{27} \mathrm{~N}_{3} \mathrm{O}_{3} \mathrm{~S}, M=497.60$, monoclinic, space group $P 1 c 1, a=9.2486(3) \AA, b=13.4063(5) \AA, c=10.1854(3) \AA, V=$ $1229.01(7) \AA^{3}, Z=2, D_{\text {calcd }}=1.354 \mathrm{Mg} \mathrm{m}^{-3}, T=296(2) \mathrm{K}, \mu=1.469$ $\mathrm{mm}^{-1}, F(000)=524$, and independent reflections 2658. The crystal data has been deposited at Cambridge Crystallographic Data Centre [CCDC No. 842895]. Copies of the data can be obtained free of charge via www.ccdc.ac.uk/conts/retrieving.html or CCDC, 12 union Road, 50 Cambridge CB2 1EZ, U.K.

(16) Martin, C.; Zhang, Y. The diverse functions of histone lysine methylation. Nat. Rev. Mol. Cell Biol. 2005, 6, 838-849.

(17) (a) Fog, C. K.; Jensen, K. T.; Lund, A. H. Chromatin-modifying proteins in cancer. APMIS 2007, 115, 1060-1089. (b) Spannhoff, A.; Sippl, W.; Jung, M. Cancer treatment of the future: Inhibitors of histone methyltransferases. Int. J. Biochem. Cell Biol. 2009, 41, 4-11.

(18) (a) Liu, F.; Chen, X.; Hassani, A. A.; Quinn, A. M.; Wasney, G. A.; Dong, A.; Barsyte, D.; Kozieradzki, I.; Senisterra, G.; Chau, I.; Siarheyeva, A.; Kireev, D. B.; Jadhav, A.; Herold, J. M.; Frye, S. V.; Arrowsmith, C. H.; Brown, P. J.; Simeonov, A.; Vedadi, M.; Jin, J. Discovery of a 2,4-diamino-7-aminoalkoxyquinazoline as a potent and selective inhibitor of histone lysine methyltransferase G9a. J. Med. Chem. 2009, 52, 7950-7953. (b) Liu, F.; Chen, X.; Hassani, A. A.; Quinn, A. M.; Wigle, T. J.; Wasney, G. A.; Dong, A.; Senisterra, G.; 
Chau, I.; Siarheyeva, A.; Norris, J. L.; Kireev, D. B.; Jadhav, A.; Herold, J. M.; Janzen, W. P.; Arrowsmith, C. H.; Frye, S. V.; Brown, P. J.; Simeonov, A.; Vedadi, M.; Jin, J. Protein lysine methyltransferase G9a inhibitors: Design, synthesis, and structure-activity relationships of 2,4-diamino-7-aminoalkoxy-quinazolines. J. Med. Chem. 2010, 53, 5844-5857. (c) Liu, F.; Lovejoy, D. B.; Hassani, A. A.; He, Y.; Herold, J. M.; Chen, X.; Yates, C. M.; Frye, S. V.; Brown, P. J.; Huang, J.; Vedadi, M.; Arrowsmith, C. H.; Jin, J. Optimization of cellular activity of G9a inhibitors 7-aminoalkoxy-quinazolines. J. Med. Chem. 2011, 54, 6139-6150. 